\section{Bendamustin plus Rituximab für NHL-Patienten}

\begin{abstract}
Patienten mit indolenten Non-Hodgkin-Lymphom (NHL) oder MantelzellLymphom (MCL) wurden über lange Zeit dem aggressiven R-CHOP-Regime behandelt. Nun konnten deutsche Hämatologen zeigen, dass das Regime Rituximab plus Bendamustin zumindest gleichwertig und besser verträglich ist.
\end{abstract}

n der prospektiv randomisierten Phase-III-Studie der deutschen Studiengruppe indolente Lymphome (StiL) wurde bei fast 550 Patienten mit niedrigmalignen NHL oder MCL in den fortgeschrittenen Stadien III oder IV der Nutzen der Zweifach-Kombination geprüft. Primäres Studienziel war eine Nicht-Unterlegenheit von Rituximab plus Bendamustin (BR; Rituximab $375 \mathrm{mg} / \mathrm{m}^{2}$ an Tag 1 alle 28 Tage, Bendamustin $90 \mathrm{mg} /$ $\mathrm{m}^{2}$ an Tag 1 und 2) im Vergleich zu RCHOP (Rituximab plus Cyclophosphamid, Doxorubicin, Vincristin und Prednison) über maximal sechs Zyklen. Das mediane Follow-up betrug 45 Monate.

Das progressionsfreie Überleben nach der Therapie war unter der Zweifachkombination mit 69,5 vs. 31,2 Monaten signifikant länger ( $p<0,0001)$. Signifikanz erreichte auch der Unterschied zwischen den beiden Gruppen beim $\mathrm{Pa}$ ramater „komplettes Ansprechen“: $40 \%$ unter BR ( $\mathrm{n}=104)$ vs. $30 \%$ unter RCHOP $(n=76 ; p=0,021)$.

Schließlich war die Behandlung mit Rituximab plus Bendamustin deutlich besser verträglich als R-CHOP - schwere unerwünschte Wirkungen waren signifikant seltener (19 vs. $29 \%$ ). So traten schwere Neutropenien (Grad 3/4) in der R-CHOP-Gruppe bei $69 \%$ der Patienten auf, in der Gruppe mit der Zweifachkombination dagegen nur bei $29 \%$ ( $\mathrm{p}$ 0,0001 ), ebenso Infektionen ( 37 vs. $50 \%$; $\mathrm{p}=0,025)$. Und: Während sich bei allen Patienten der Chemotherapie-Gruppe eine Alopezie entwickelte, war das bei keinem Patienten unter der weniger aggressiven Therapie der Fall.

Fazit: Aufgrund dieser Studienergebnisse empfehlen die Hämatologen um Mathias J. Rummel vom Universitätsklinikum Gießen die Kombination Rituximab plus Bendamustin bei Patienten mit indolenten NHL und MCL als Erstlinientherapie. Wie es in einem Kommentar zur Studie im „Lancet“ heißt, ist jedoch noch unklar, wie die Kombination den Langzeitverlauf der NHL beeinflussen wird.

Peter Leiner

Rummel MJ et al. Bendamustine plus rituximab versus $\mathrm{CHOP}$ plus rituximab as first-line treatment for patients with indolent and mantle-cell lymphomas: An open-label, multicentre, randomised, phase 3 non-inferiority trial. Lancet. 2013;381(9873):1203-10.

Kommentar von Dr. Abenhardt: Die Ergebnisse der StiL NHL1-Studie sind in mehrfacher Hinsicht eindrucksvoll:

1. Es bedeutet so ziemlich die höchstmögliche wissenschaftliche Wertschätzung, in einer Plenarsitzung des jährlichen ASCO-Meetings (Chicago 2012), zudem als Deutscher und im hämatoonkologischen Bereich, Resultate einer deutschen multizentrischen Therapiestudie präsentieren zu dürfen, von der mehrfachen Publikation im JCO ganz zu schweigen.

2. Renommierte amerikanische Kommentatoren haben diese Resultate ohne Abstriche gewürdigt und BR zum neuen Therapiestandard bei indolenten Lymphomen erklärt. In den wichtigen internationalen Leitlinien von ASCO, NCCN und ESMO ist das BR-Protokoll als therapeutischer Standard bei indolenten Lymphomen etabliert.

3. Planung und Durchführung der StiL NHL1-Studie verliefen hochprofessionell mit klarem, praxisorientiertem Konzept ohne wissenschaftliche Überfrachtung. Durch eine vorbildliche engagierte Zusammenarbeit von stationären und niedergelassenen Zentren konnte in Rekordzeit rekrutiert werden. Das mediane Alter der Patienten von 64 Jahren belegt die große Praxisrelevanz dieser Studie.

4.Aus strategischen Überlegungen hat sich die Studie nicht nur auf follikuläre Lymphome (FL) beschränkt, sondern auch auf andere indolente Lymphome wie MCL und MarginalzonenLymphome. In allen Entitäten zeigte das BR-Protokoll bis heute (sieben Jahre!) deutliche Vorteile gegenüber R-CHOP: OR 93 vs. $91 \%$, CR 40 vs. $30 \%$, PFS 70 vs. 31 Monate, OS (5 Jahre) 76 vs. $60 \%$. Auch MCL und MW sowie höhere FLIPI-Stadien haben signifikant profitiert, besonders deutlich die schwer therapierbaren Mantelzell-Lymphome. Angesichts der Plateaubildung nach sieben Jahren im BR-Arm wird sogar schon über eine bislang undenkbare - Möglichkeit einer Heilung spekuliert.

5. Die Nebenwirkungen waren im BRArm bezüglich Neuropathie, Myelosuppression und Alopezie deutlich

„Über eine - bislang undenkbare Heilung wird angesichts einer Plateaubildung nach sieben Jahren spekuliert."

günstiger. Dass die seinerzeit - auch von uns erfahrene - gute subjektive Verträglichkeit (Akutreaktionen, ANE) jetzt nicht mehr in dieser Form reproduzierbar ist, lässt an eine chemische Veränderung denken und muss weiter eruiert werden.

6. Ein Wermutstropfen bleibt allerdings zu erwähnen: Die StiL NHL1 war statistisch auf Nichtunterlegenheit ausgelegt. Diese wurde zweifelsfrei bewiesen und BR - schon wegen der besseren Verträglichkeit - als klare Option etabliert. R-CHOP wurde damit jedoch (als jahrzehntelanger unschlagbarer) Standard nicht abgelöst. Insbesondere auch bei hochmalignen NHL bleiben - wie in der medizinischen Wissenschaft üblich - offene Fragen für weitere Studien.

PS: Bendamustin war eines der Hauptzytostatika der ehemaligen DDR und insbesondere bezüglich seiner zuverlässigen Wirkung nicht nur bei lymphatischen Neoplasien und guter Verträglichkeit bekannt. Durch seine bifunktionelle Wirkungsweise ist Bendamustin eines der wenigen Zytostatika ohne bisher bekannte Resistenzentwicklung.

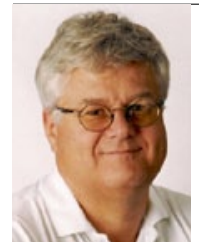

Dr. med. Wolfgang Abenhardt, MOP Elisenhof MVZ, München w.abenhardt@onkologieelisenhof.de 